\title{
Validation of JEFF-3.3 and ENDF/B-VIII.0 nuclear data libraries in ANSWERS codes
}

\author{
Tim Ware ${ }^{1, *}$, David Hanlon ${ }^{1}$, Glynn Hosking ${ }^{1}$, Ray Perry ${ }^{1}$, and Simon Richards ${ }^{1}$ \\ ${ }^{1}$ Wood, Kings Points House, Queen Mother Square, Poundbury, Dorchester, Dorset, DT1 3BW, UK
}

\begin{abstract}
The JEFF-3.3 and ENDF/B-VIII.0 evaluated nuclear data libraries were released in December 2017 and February 2018 respectively. Both evaluations represent a comprehensive update to their predecessor evaluations. The ANSWERS Software Service produces the MONK ${ }^{\circledR}$ and MCBEND Monte Carlo codes, and the WIMS deterministic code for nuclear criticality, shielding and reactor physics applications. MONK and MCBEND can utilise continuous energy nuclear data provided by the BINGO nuclear data library and MONK and WIMS can utilise broad energy group data (172 group XMAS scheme) via the WIMS nuclear data library. To produce the BINGO library, the BINGO Pre-Processor code is used to process ENDF- 6 format evaluations. This utilises the RECONR-BROADR-PURR sequence of NJOY2016 to reconstruct and Doppler broaden the free gas neutron cross sections together with bespoke routines to generate cumulative distributions for the $\mathrm{S}(\alpha, \beta)$ tabulations and equi-probable bins or probability functions for the secondary angle and energy data.

To produce the WIMS library, NJOY2016 is again used to reconstruct and Doppler broaden the cross sections. The THERMR module is used to process the thermal scattering data. Preparation of data for system-dependent resonance shielding of some nuclides is performed. GROUPR is then used to produce the group averaged data before all the data are transformed into the specific WIMS library format.

The MONK validation includes analyses based on around 800 configurations for a range of fuel and moderator types. The WIMS validation includes analyses of zero-energy critical and sub-critical, commissioning, operational and post-irradiation experiments for a range of fuel and moderator types. This paper presents and discusses the results of MONK and WIMS validation benchmark calculations using the JEFF-3.3 and ENDF/BVIII.0 based BINGO and WIMS nuclear data libraries.
\end{abstract}

\section{Introduction}

The JEFF-3.3 evaluated nuclear data library was released in December 2017 and the ENDF/B-VIII.0 library was released in February 2018. These represent the of culmination many years of measurement and evaluation.

The ANSWERS Monte Carlo criticality code MONK [1] can be run using multi-group or continuous energy nuclear data. For continuous energy mode, MONK uses the BINGO collision processor and continuous energy data library. The BINGO collision processor and library is also used with MONK's sister code, MCBEND, for shielding and dosimetry calculations. The multi-group library used by MONK is the WIMS 172-group library used in the ANSWERS deterministic neutronics code, WIMS [2].

For the BINGO library, it is necessary to transform the evaluated data to a form that allows efficient Monte Carlo sampling. This is done using the BINGO Pre-Processor (BPP) code. The BPP uses the NJOY code to process the cross sections while the secondary angle and energy data are transformed by the BPP. For the WIMS library, NJOY is further used to produce the group averaged data for both the cross sections and emitted data.

*e-mail: tim.ware@woodplc.com

\section{BINGO Library Production}

MONK uses continuous energy nuclear data with cross sections represented as a smoothly varying function of energy and tabulated on an energy grid that allows a maximum interstitial error in the cross section of $0.1 \%$. The evaluated data are processed using the BINGO preprocessor (BPP). The BPP currently uses NJOY2016 [3] to generate the point cross sections via the RECONR and BROADR modules for reconstruction and Doppler broadening to the required accuracy and temperature. The remaining processing is performed internally by the BPP. The secondary angle and energy data for emitted neutrons are transformed into equi-probable bins, probability functions or sampled from algorithms defined in the ENDF-6 format [4]. The $\mathrm{S}(\alpha, \beta)$ thermal scattering data are transformed to ease bivariate sampling in momentum and energy, allowing MONK to generate secondary energy neutron emission data during the run.

In the resolved resonance range, the continuous energy representation is adequate to model resonance shielding effects. In the unresolved resonance range, a small number of nuclides require special processing. Here, 1/128 lethargy width group data are generated at infinite dilution and at a level of resonance self-shielding applicable to thermal reactors. They are then collected in pairs, to 
form two sub-groups, and equations applied that preserve the infinite dilute and shielded cross sections across the pair of groups. These equations increase the infinite dilute paired cross section by a fixed amount and apply this cross section in one of the original groups. The cross section in the other original group is reduced by that level. This effectively moves the resonance peaks to one of the subgroups forcing a flux depression dependent on the level of shielding. In BINGO, the sub-group cross sections are refined by allowing variable lethargy sub-group widths and slope on the top of the histogram. The sub-group data can be sampled using standard Monte Carlo techniques.

Library data are generated at a standard set of temperatures including 200, 273.15, 293.6, 500, 1000, 1500 and $2000 \mathrm{~K}$, plus higher temperatures up to $80,000 \mathrm{~K}$. Data for intermediate temperatures are calculated by MONK at run time using an accelerated Doppler broadening method. This method cannot be applied to bound thermal scattering data or unresolved resonance sub-group data and for these, interpolation is used. Bound thermal scattering data are stored at the temperatures present in the $\mathrm{S}(\alpha, \beta, \mathrm{T})$ data in the relevant ENDF-6 evaluations and in MONK version 11 (in development), stochastic interpolation of the secondary bound scattering data is used. For the unresolved resonances, the BPP generates cross sections at a set of intermediate temperatures such that linear interpolation can be applied to within a given tolerance. The overall library generation is visualised in Figure 1.

\section{WIMS Library Production}

The nuclear data library, used by the deterministic reactor physics code WIMS contains cross sections in the form of group averaged data. The standard energy group scheme is currently the XMAS 172 group scheme [2]. The majority of the data on the library are reaction cross sections but burnup data (decay data, fission product yields, burnup chains), heating data and other data for specific applications are also included.

The majority of the cross section data are generated using NJOY2016 [3]. In common with the BINGO library, RECONR and BROADR are used to reconstruct and Doppler broaden the cross sections and PURR is used to produce self-shielded cross sections in the unresolved resonance range. In addition, THERMR is used to generate thermal scattering data and GROUPR to generate self-shielded multi-group cross sections and groupto-group scatter matrices. The weighting function used by GROUPR for the group condensation for all nuclides comprises a thermal Maxwellian spectrum below $0.2 \mathrm{eV}$, a $1 / \mathrm{E}$ weighting in the intermediate range and a Maxwellian fission spectrum above $820 \mathrm{keV}$. For nuclides with resonance shielding, the group dependent resonance integrals are generated by NJOY using a slowing down flux calculated using a mixture of the resonant nuclide and hydrogen.

In the resonance range, infinite dilution data, at 293.16 K, are held for the majority of nuclides. For significant resonant nuclides, resonance integrals per unit lethargy are stored for a range of background cross sections $\left(\sigma_{p}\right)$ and temperatures. WIMS calculates the $\sigma_{p}$ for a particular reactor system and then interpolates between the fixed library values to obtain the resonance integrals for that system and hence the appropriate level of shielding. A small number of nuclides, $\mathrm{Fe}, \mathrm{Cr}$, Ni and $\mathrm{Zr}$, use "pre-shielded" cross sections, generated via reaction rates calculated using the ECCO code [2].

In the thermal energy region, below $4 \mathrm{eV}$, data for actinides, shielded nuclides and selected other nuclides are calculated using a free gas model, at a set of standard temperatures: 293.16, 573.16, 973.16, 1473.16 and 2973.16 K. WIMS uses a linear interpolation scheme to determine cross sections between these temperatures. Bound thermal scattering data are generated at the temperatures provided in the relevant $\mathrm{S}(\alpha, \beta, \mathrm{T})$ evaluated file. Data for all other nuclides are at 293.16 K only.

The overall library generation is visualised in Figure 1.

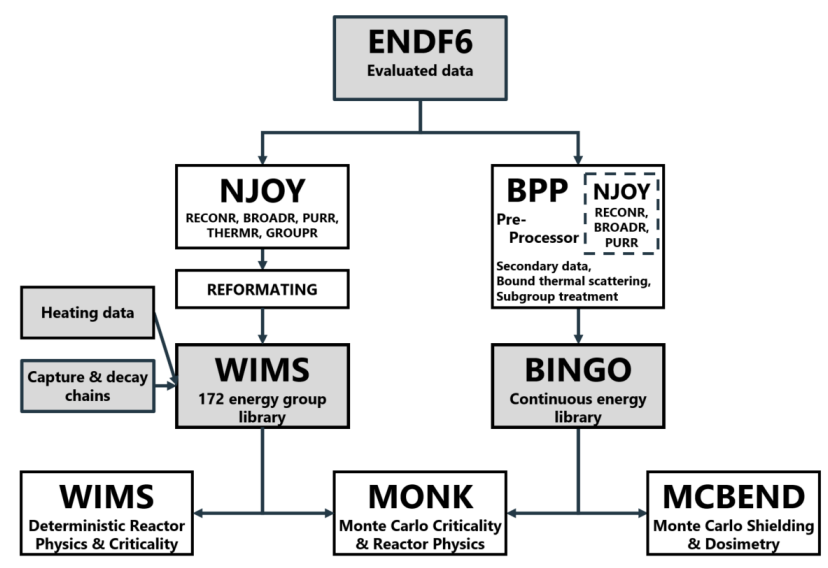

Figure 1. BINGO and WIMS library production route.

\section{MONK Validation}

The MONK validation database comprises "Tier 1" cases, consisting of around 800 configurations from 95 experiments mostly based on the ICSBEP handbook [5]. These cases are independently reviewed and are the primary validation for MONK. They are used with the MONK categorisation scheme which categorises every application case based on six properties tallied during tracking in order to identify validation cases that are comparable with the application case. Additionally, there exists "Tier 2" cases (more than 1000 configurations) which are yet to be independently reviewed and included in the categorisation scheme. The majority of the Tier 1 cases have been performed for the results presented here.

The systems modelled comprise uranium, plutonium and mixed $\mathrm{U} / \mathrm{Pu}$, in the form of metals, solutions and compounds. Each case was run four times and the results of each combined to achieve an overall convergence of $50 \mathrm{pcm}$.

To quantify the agreement between the MONK calculation and the experimental k-effective, The reduced $\chi_{\text {red }}^{2}$ 
is calculated as;

$$
\chi_{\text {red }}^{2}=\frac{1}{N} \sum_{i=1}^{N} \frac{\left(k_{\text {MONK }}-k_{\text {benchmark }}\right)^{2}}{\Delta\left(k_{\text {MONK }}\right)^{2}+\Delta\left(k_{\text {benchmark }}\right)^{2}}
$$

where the $\Delta \mathrm{s}$ are the stochastic uncertainty and experimental uncertainty respectively.

Figures 2 through 9 show the cumulative $\chi_{\text {red }}^{2}$ for eight of the fissile species and material combinations. The six plutonium compound cases are not shown as five of these are configurations of one experiment and show a $2 \%$ bias in $\mathrm{C} / \mathrm{E}$ values for all four libraries. In each figure, the cases are ordered with respect to their average $\chi^{2}$ such that the worst performing cases are on the right of the plot.

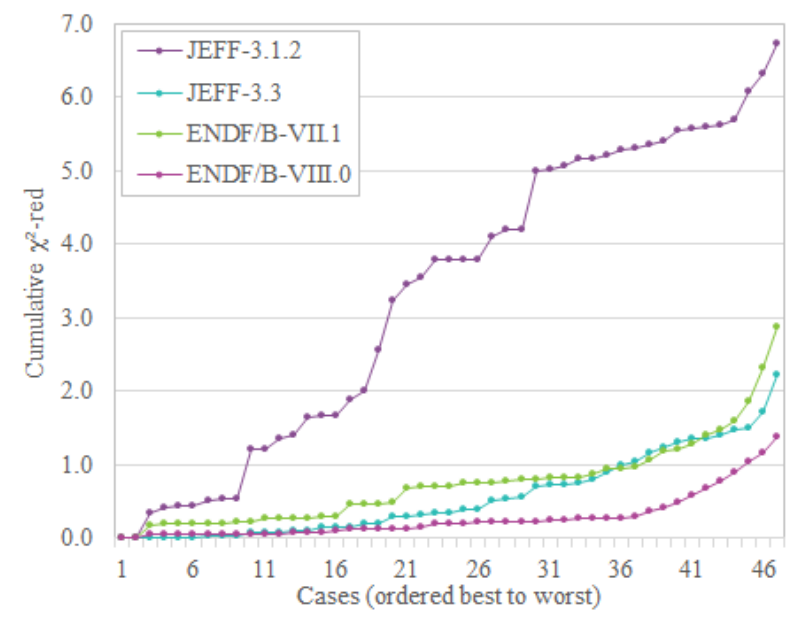

Figure 2. Cumulative $\chi_{\text {red }}^{2}$ for 47 uranium metal cases.

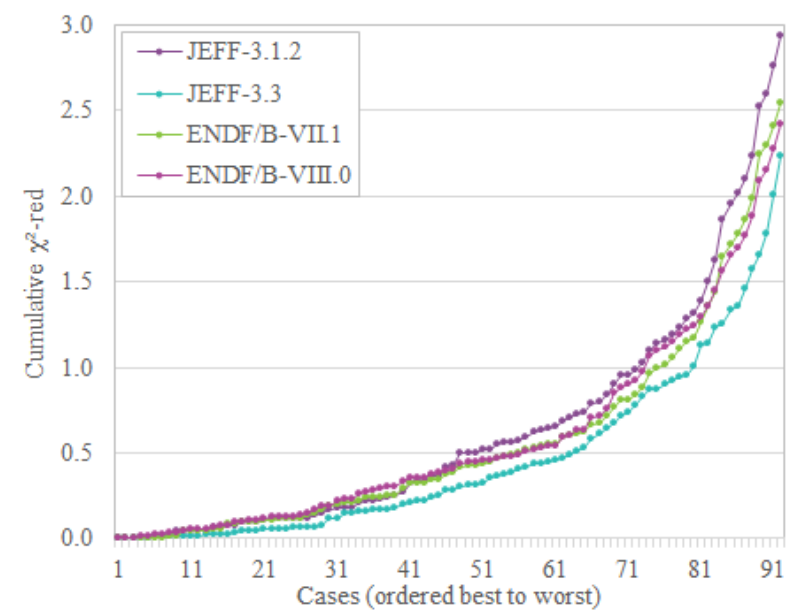

Figure 3. Cumulative $\chi_{\text {red }}^{2}$ for 92 uranium solution cases.

For the uranium metal cases, JEFF-3.3 and ENDF/BVIII.0 show an overall improvement over their predecessors. For uranium solutions, the four evaluations show similar performance, with JEFF-3.3 giving a slight improvement and the ENDF/B evaluations giving very similar results. For uranium compounds, JEFF-3.3 performs worse than the other evaluations. ENDF/B-VIII.0 is a slight improvement on ENDF/B-VII.1.

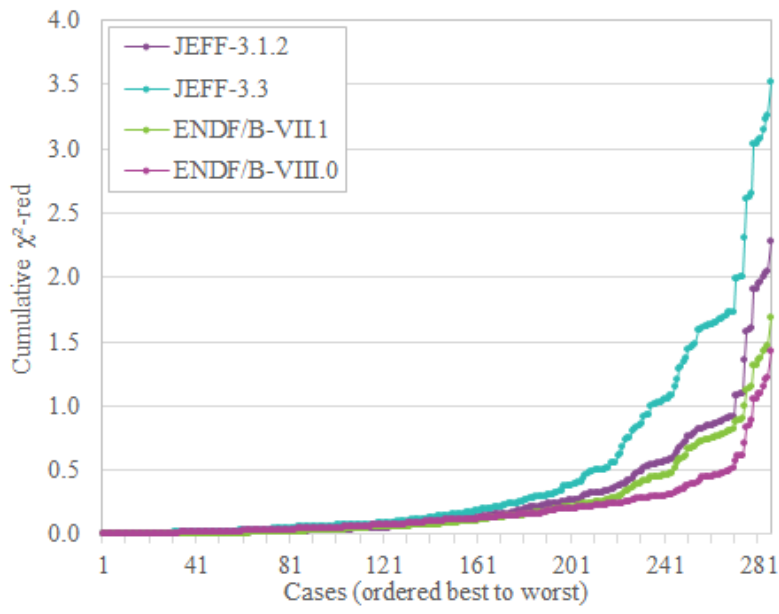

Figure 4. Cumulative $\chi_{\text {red }}^{2}$ for 286 uranium compound cases.

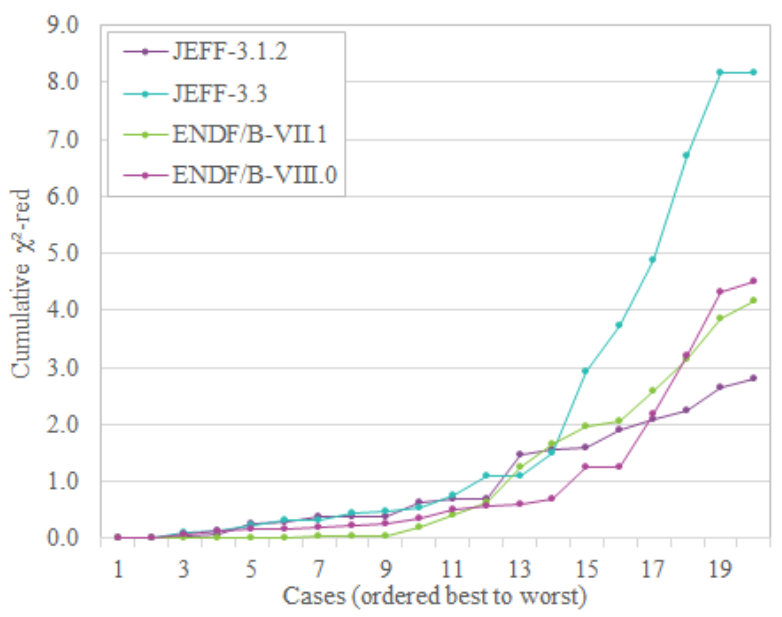

Figure 5. Cumulative $\chi_{\text {red }}^{2}$ for 20 plutonium metal cases.

For plutonium metals, the ENDF/B evaluations give similar performance, with JEFF-3.1.2 being slightly better and JEFF-3.3 being significantly worse. For plutonium solutions, ENDF/B-VII.1 is significantly worse than the other three evaluations which have similar performance.

For mixed U/Pu metals, ENDF/B-VII.1 is significantly the worst, with JEFF-3.3 and ENDF/B-VIII.0 performing similarly and JEFF-3.1.2 performing the best. For mixed U/Pu solutions, JEFF-3.1.2 and ENDF/B-VII.1 perform similar to one another and slightly better than JEFF3.3 and ENDF/B-VIII.0. For mixed U/Pu compounds, all evaluations perform well and similarly for the majority of cases with poorer performance for just seven cases with ENDF/B-VII.1 being worse than the others.

\section{WIMS Validation}

Three sets of cases from the WIMS validation database are presented here. WIMS calculates problem-dependent macroscopic cross sections starting from the 172-group WIMS nuclear data library. The radiation transport equation can be solved by one of several deterministic methods 


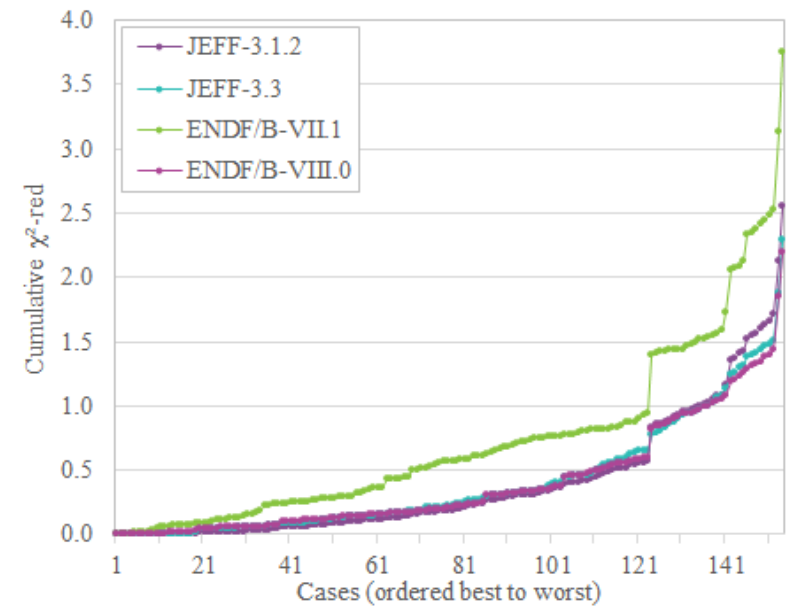

Figure 6. Cumulative $\chi_{\text {red }}^{2}$ for 154 plutonium solution cases.

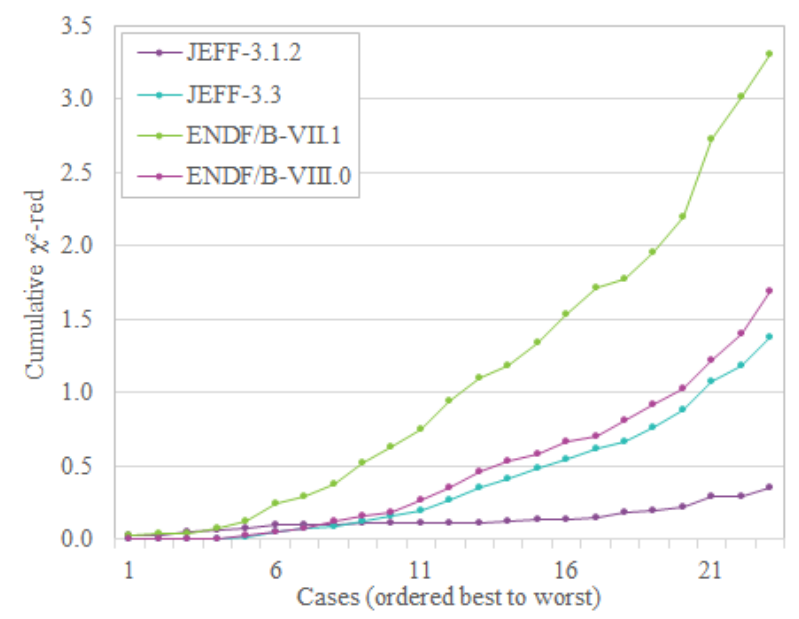

Figure 7. Cumulative $\chi_{\text {red }}^{2}$ for 23 mixed metal cases.

including the Method-of-Characteristics. Alternatively, an embedded version of the MONK code provides a Monte Carlo solution.

Note that the tables in this section abbreviate the evaluation names, "J" = JEFF, "E" = ENDF/B.

\subsection{Brookhaven Experiments}

These cases are based on a specification provided in [6]. Resonance shielding is performed via the equivalence method. The collision probabilities for the annular geometry are calculated by Gaussian Integration.

The fuel pins in these lattices were $3 \% / \mathrm{o}$ enriched $\mathrm{UO}_{2}$ with stainless steel cladding. The moderator was light water. Experiments were made with one batch of fuel containing no boron and with a second batch of fuel with dissolved boric acid in a range of concentrations. The $\frac{V_{m}}{V_{f}}$ ratio in each configuration was between 1.3 and 4.1. Table 1 gives the k-effectives calculated by WIMS for each configuration and each evaluated library.

The experimental k-effective is assumed to be unity for all configurations. Therefore, all the evaluations overpre-

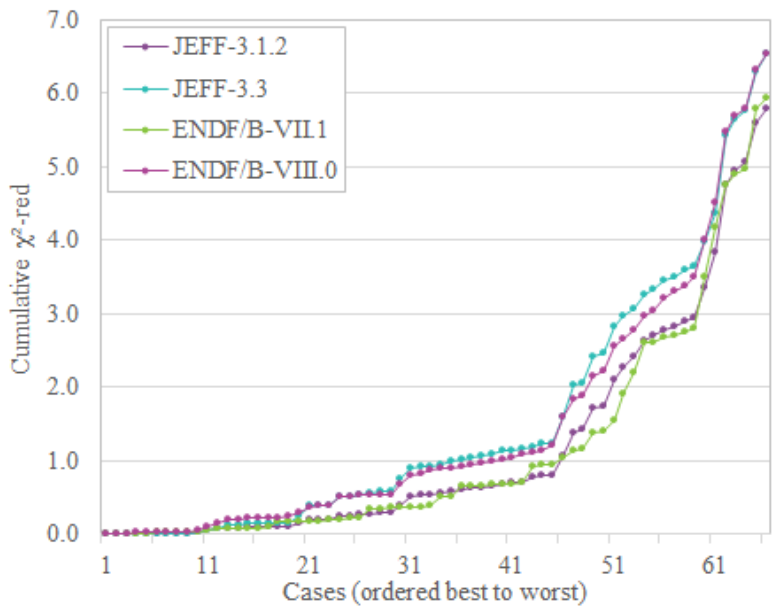

Figure 8. Cumulative $\chi_{\text {red }}^{2}$ for 66 mixed solution cases.

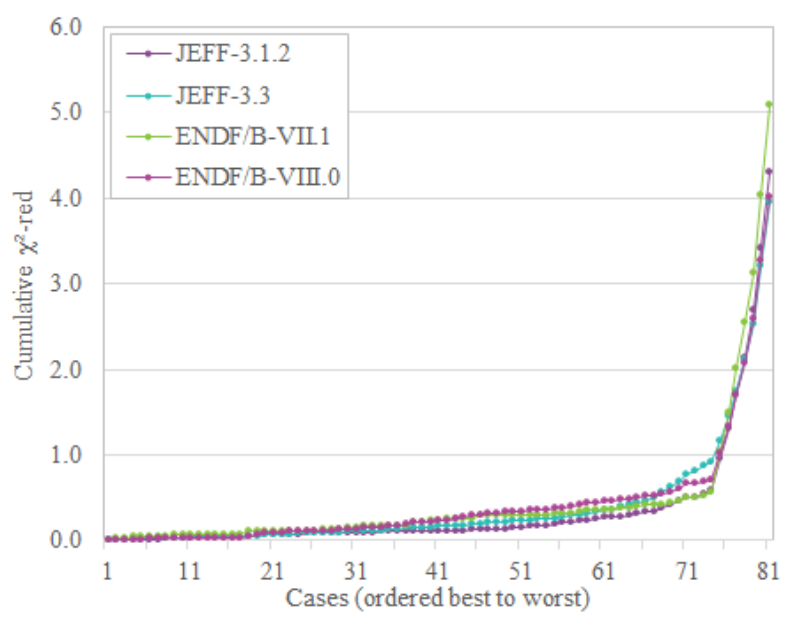

Figure 9. Cumulative $\chi_{\text {red }}^{2}$ for 81 mixed compound cases.

dict k-effective by around $400 \mathrm{pcm}$. For the higher moderation configurations, all four evaluations are in good agreement. For the lower moderation configurations, the JEFF3.3 results are higher than the other evaluations.

\subsection{DIMPLE Experiments}

These cases are based on a specification provided in [5] (LCT-048,055,063). The resonance shielding was determined using the subgroup method. The flux solution was obtained via the Method-of-Characteristics.

The fuel pins in these lattices was $3 \%$ enriched $\mathrm{UO}_{2}$ with stainless steel cladding. The moderator was light water. The six configurations differed in the number of fuel pins, pin pitch and critical water height. These configurations have high neutron leakage. The experiments were performed at room temperature. Table 2 gives the keffectives calculated by WIMS for each configuration and each evaluated library.

All four evaluations underpredict k-effective for the S01 case by around $400 \mathrm{pcm}$. The evaluations predict k-effective for the S06 cases within $+500 \mathrm{pcm}-$ $300 \mathrm{pcm}$. There is very good agreement between the 
Table 1. Brookhaven calculated k-effectives.

\begin{tabular}{ccc|cccc}
\hline No. & $\mathrm{V}_{\mathrm{m}}: \mathrm{V}_{\mathrm{f}}$ & Boron & $\mathrm{J} 312$ & $\mathrm{~J} 33$ & $\mathrm{E} 71$ & $\mathrm{E} 80$ \\
\hline 1 & 1.3 & - & 1.0094 & 1.0104 & 1.0083 & 1.0081 \\
2 & 1.6 & - & 1.0035 & 1.0043 & 1.0023 & 1.0025 \\
3 & 2.1 & - & 1.0046 & 1.0050 & 1.0033 & 1.0038 \\
4 & 2.9 & - & 1.0070 & 1.0071 & 1.0058 & 1.0066 \\
5 & 4.1 & - & 1.0049 & 1.0047 & 1.0041 & 1.0051 \\
6 & 1.3 & Mid & 1.0050 & 1.0063 & 1.0043 & 1.0041 \\
7 & 1.3 & High & 1.0016 & 1.0034 & 1.0014 & 1.0011 \\
8 & 1.6 & Low & 1.0056 & 1.0066 & 1.0048 & 1.0049 \\
9 & 1.6 & High & 1.0037 & 1.0049 & 1.0032 & 1.0033 \\
10 & 1.6 & High & 1.0028 & 1.0042 & 1.0026 & 1.0026 \\
11 & 2.1 & Low & 1.0053 & 1.0059 & 1.0045 & 1.0049 \\
12 & 2.1 & Mid & 1.0035 & 1.0043 & 1.0031 & 1.0034 \\
13 & 2.1 & High & 1.0012 & 1.0022 & 1.0011 & 1.0013 \\
14 & 2.8 & Low & 1.0051 & 1.0053 & 1.0044 & 1.0051 \\
15 & 2.8 & Mid & 1.0035 & 1.0038 & 1.0033 & 1.0038 \\
16 & 2.8 & Mid & 1.0015 & 1.0019 & 1.0015 & 1.0019 \\
17 & 4.1 & Low & 1.0048 & 1.0045 & 1.0044 & 1.0053 \\
18 & 4.1 & Low & 1.0047 & 1.0044 & 1.0045 & 1.0052 \\
19 & 4.1 & Low & 1.0022 & 1.0020 & 1.0023 & 1.0029 \\
20 & 4.1 & Mid & 1.0038 & 1.0036 & 1.0044 & 1.0047 \\
\hline
\end{tabular}

Table 2. DIMPLE calculated k-effectives.

\begin{tabular}{lcc|cc}
\hline Config. & J312 & J33 & E71 & E80 \\
\hline S01 & 0.9936 & 0.9951 & 0.9934 & 0.9921 \\
S06A & 1.0030 & 1.0053 & 1.0031 & 1.0016 \\
S06B & 1.0004 & 1.0025 & 1.0004 & 0.9988 \\
S06C/0 & 1.0017 & 1.0037 & 1.0015 & 0.9999 \\
S06C/8 & 0.9983 & 0.9999 & 0.9982 & 0.9968 \\
S06C/10 & 0.9987 & 1.0009 & 0.9989 & 0.9972 \\
\hline
\end{tabular}

JEFF-3.1.2 and ENDF/B-VII.1 evaluations. JEFF-3.3 is around $200 \mathrm{pcm}$ higher than this pair and ENDF/B-VIII.0 is around $150 \mathrm{pcm}$ lower than this pair.

\subsection{KRITZ-2 Experiments}

The KRITZ experiments [7] (KRITZ-LWR-RESR001,002,003) include a series of criticality experiments on light water moderated lattices with uranium and mixed oxide fuel rods, at room temperature ("cold") and at temperatures up to $500 \mathrm{~K}$ ("hot"). The KRITZ-2 series analysed here includes experiments with three core configurations;

- 2.1 and 2.13 were $\mathrm{UO}_{2}\left(1.86 \mathrm{w} / \mathrm{o}{ }^{235} \mathrm{U}\right)$ fuel pins in a square lattice (different number and pin pitch).

- 2.19 was $\mathrm{MOX}\left(\mathrm{UO}_{2}+1.50 \mathrm{w} / \mathrm{PuO}_{2}, 0.16^{\mathrm{w}} / \mathrm{o}{ }^{235} \mathrm{U}\right.$ in $\mathrm{U}$, $91.41 \% /{ }^{239} \mathrm{Pu}$ in $\mathrm{Pu}$ ) fuel pins, also in a square lattice.

All configurations were moderated and reflected by light water containing a boron poison. Criticality was attained by controlling the boron content in the water, and by adjusting the water level. For the data validation, the WIMS data library was used with the resonance shielding determined by the equivalence method. The in-built MONK module was used to predict k-effective via the Monte Carlo method with a stochastic uncertainty of 0.0004 . The uncertainty in the benchmark model $\mathrm{k}$ effective ranges from 0.0020 to 0.0030 .
Table 3. KRITZ-2 C/E results.

\begin{tabular}{ccc|cccc}
\hline Config. & Temp. & Fuel & J312 & J33 & E71 & E80 \\
\hline 2.1 & cold & $\mathrm{UO}_{2}$ & 0.9945 & 0.9963 & 0.9934 & 0.9930 \\
2.1 & hot & $\mathrm{UO}_{2}$ & 0.9894 & 0.9928 & 0.9893 & 0.9891 \\
2.13 & cold & $\mathrm{UO}_{2}$ & 0.9971 & 0.9992 & 0.9980 & 0.9982 \\
2.13 & hot & $\mathrm{UO}_{2}$ & 0.9946 & 0.9979 & 0.9941 & 0.9955 \\
2.19 & cold & $\mathrm{MOX}$ & 0.9928 & 0.9922 & 0.9940 & 0.9911 \\
2.19 & hot & MOX & 0.9942 & 0.9891 & 0.9938 & 0.9921 \\
\hline
\end{tabular}

Table 3 gives the $\mathrm{C} / \mathrm{E}$ values for WIMS calculated $\mathrm{k}$ effectives against the experimental k-effectives. The experimental k-effectives are between 1.001 and 1.008.

For the $\mathrm{UO}_{2}$ cases, JEFF-3.1.2 and the ENDF/B evaluations are in very good agreement with one another. JEFF3.3 predicts higher k-effectives which are closer to the experimental values. There is more spread in the predictions for the MOX cases, with ENDF/B-VII.1 giving the best agreement with experiment. JEFF-3.3 performs relatively poorly for the hot MOX case. Overall, the majority of the calculated k-effectives are within $3 \sigma$ of the experimental results, with all within $4.1 \sigma$.

\section{Conclusions}

Continuous energy (BINGO) and multi-group (WIMS) nuclear data libraries have been produced for the ANSWERS Monte Carlo code MONK and the deterministic code WIMS, based on the JEFF-3.3 and ENDF/B-VIII.0 evaluations. The new BINGO and WIMS libraries have been used in calculations for a range of MONK Monte Carlo and WIMS deterministic code validation cases and compared with existing libraries based on JEFF-3.1.2 (reported at ND2013) and ENDF/B-VII.1.

Overall, all four evaluations perform well against experiment though some evaluations perform better for specific experimental configurations. This highlights the need for users to consider the results of validation cases that are comparable with their specific applications (using the MONK categorisation scheme, or similar system).

Some specific differences have been identified in JEFF-3.3 relative to JEFF-3.1.2. The change in ${ }^{235} \mathrm{U} \bar{v}$ reduces k-effective but the impact of the change in the thermal cross section is dependent on the thermal neutron spectrum (itself dependent on temperature and fuel poisoning) and the neutron leakage, leading to a range of effects on k-effective. A distinct trend is the increase in keffective for high temperature cases. However, as demonstrated by the KRITZ- $2 \mathrm{UO}_{2}$ cases, JEFF- 3.3 can give improved agreement with experiment. In contrast, the change in the ${ }^{239} \mathrm{Pu}$ fission cross section leads to an underprediction of k-effective at raised temperatures, as shown in the KRITZ-2 MOX fuel case.

\section{References}

[1] S. D. Richards et al., Annals of Nuclear Energy 82, 63-73 (2015)

[2] B. Lindley et al. Proceedings of International Congress on Advances in Nuclear Power Plants, Nice, France (2015) 
[3] A. C. Kahler (ed.), LA-UR-17-20093 (2016)

[4] A. Trkov, M. Herman and D. A. Brown (eds.), CSEWG Document ENDF-102, Report BNL-2032182018-INRE (2018)

[5] OECD Nuclear Energy NEA/NSC/DOC(95)3 (2018)
[6] OECD Nuclear Energy Agency, NEACRP-U-190 (1977)

[7] OECD Nuclear Energy Agency, IRPHE2018HANDBOOK (2018) 